\title{
SH
}

11

$\mathrm{A}_{43}$

1910

IRTMENT OF COMMERCE AND LABOR BUREAU OF FISHERIES

FISCAL REGULATIONS

governing

Employments in the Field

Effeative February 1, 1910

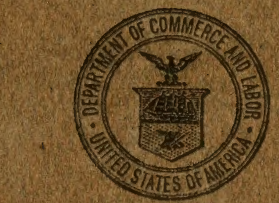

WASHINGTON

Government Printing Office

1910

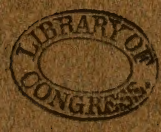




$$
\text { ชิ }
$$








\title{
DEPARTMENT OF COMMERCE AND LABOR
} BUREAU OF FISHERIES

\section{FISCAL REGULATIONS}

\author{
governing
}

Employments in the Field

Effective February 1, 1910

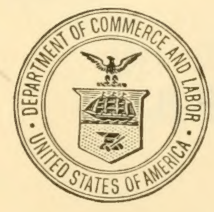

WASHINGTON

Government Printing Office

1910 


$$
\begin{aligned}
& S H 11 \\
& A 4^{3} \\
& 1910
\end{aligned}
$$

$$
\begin{gathered}
\text { FEB } \& 1910 \\
\text { WOF }
\end{gathered}
$$


February 1, 1910.

The following regulations are hereby promulgated for the guidance of officers and employees properly authorized to perform field service under the Bureau of Fisheries.

Geo. M. Bowers, Commissioner of Fisheries.

Approved:

Benj. S. Cable, Acting Secretary.

(3) 

1. Expense accounts not prepared on voucher blanks in conformity with these instructions will be returned for correction, as the Comptroller of the Treasury holds that accounts of field employees can not be altered in any particular in the administrative office.

2. Each employee engaged in field work will be furnished a copy of these regulations, telegraph identification cards, stationery, and the following-described jorinted forms: government requests for transportation, vouchers of the kinds required, subvouchers, and, if necessary, government bills of lading and reports of temporary labor employed. Further supplies may be obtained by making requisition on the Bureau of Fisheries.

The instrurtions printed on these blanks are to be carefully followed.

3. Every employee acting under travel orders or engaged in the field service of the Bureau of Fisheries is required to keep himself provided with funds sufficient for all of his current expenses. If loss of time results from lack of funds, such time will be charged to the annual leave of the employee, or deducted from his salary, and no subsistence charge will be allowed therefor.

4. Every employee must keep a memorandum of expenditures, noting each item at the time the expense is incurred, together with the date. 
5. At the termination of the term of service of an employee, unused govermment requests for transportation, telegraph identification cards, bills of lading, and all government property in his possession must be arcounted for to the Bureau before his final voucher will be approved for settlement.

\section{TRAVELING EXPENSES.}

6. All travel should be by the shortest practicable route and without any unusial or unnecessary delay.

Proper and legitimate traveling expenses are those "usual and essential" to the comfort of travelers, and include the following:

7. Fares upon railroads, steamboats, stages, or other usual means of transportation.-Charges for fares on steamboats should show whether meals and berth are included. The purchase of mileage books, commutation tickets, or the acceptance of rebates, in any form, on fares is prohibited, but through, limited, or round-trip tickets must be procured whenever possible and when not used the reason must be stated.

8. Government requests for transportation must be used where the cost of transportation involved is more than two dollars, and, when used, that fact should be indicated in the account, as shown in sample account, with the date of issue. If it is impracticable in any case to use such requests, the reasons must be stated. The number of the travel order must be entered on the face of the request.

9 . The coupon of the government request for transportation properly filled out, including the number of the travel order, or, if not numbered, the date of such order, is to be forwarded, after the completion of the journey for which the transportation was furnished, to the Bureau of Fisheries, and when a request is issued for sleeping-car or parlor-car-accommodations the conductor's checks for berths or seats are to be forwarded with the coupon. 
10. Travel on limited trains requiring extra fares will not be allowed except when al)-olutely necesary. In this case and when unusual route or expensive neans of transportation are charged for, an explanatory statement setting forth the necesity for the expenses must accompany the account, and, if approved by the commisioner of Fisheries, such charges will be allowed. 'The ronte of an employee should be carchully arranged to aroid diuplication in travel.

11. Points between which travel is periormed must be indicated in the arcount. Junction points must also be shown.

12. Payment of fares to conductors of trains must be shown by subrouchers, and if the oinision to purchase tickets is not satisfactorily explained the extra cost will not be allowed.

Unused coupons of railroad tickets must he forwarded to the Bureau for redemption.

13. Sleeping-car fares (one double berth), fares for parlor-car seats on trains, and for customary stateroom accommodations on boats.--Pasengers' ('oupons isued as receipts for berthe in sleeping cars and seats in parlor car: must accompany the account as subvouchers when paid for in cash instead of with govermment requests for transportation.

14. Porter fee not exceeding 25 ents on each sleeping or parlor car. If one car is used for an extended period, a fee of 25 cents will be allowed for each $2+1$ hours or fraction thereof. Tips generally will not be allowed. Baggage porters' fees will be allowed where the performance of service is shown.

15. Street-car fares, etc., between depots and hotels and other necessary points; and transferring and checking baggage at depots.-Charges for street-car fares should show points between which travel is made. Charges for personal transfer and baggage will be closely 19405-10-2 


\section{8}

scrutinized, and if regarded excessive, as compared with the charges of other employees engaged in similar work, an explanation will be required. Charges for omnibus, transfer coach, or cab will not be allowed if street-railway cars were available. All such expenses should be fully explained.

16. Reasonable hotel accommodations.-Hotel charges if involving lodging must be supported by subrouchers shorring the regular per diem rate for lodging, for board, or for both, as the case may be, together with the times between which the same were furinshed. Charges for meals and lodging must be it emized on the subroucher, e. g., "From supper July 3 to supper July 5, inclusire, 2 ? days, at $\$ 2, \$ 4.50 . "$

17. Meals must be designated in accounts as breakfast, dinner, and supper, and the places at which meals are taken must be stated, excepting those on dining cars, when they must be stated in the account as "en route." Subvouchers are not required for detached meals.

1S. No allowance for board or subsistence will be made in case of aroidable detentions not required in the performance of duties designated in orders.

19. No allowance will be made for expenses incurred for meals or lodging at the place where the cmploree resides.

20. Bills for board and subsistence must be praid by the employee incurring the expense and not forwarded to the Bureau for settlement.

21. Livery and other special transportation will be allowed when there are no regular means, such as railroads, steamboats, or stages; al:o when the use of same results in the saring of money. Subrouchers showing the neressity for the hire of such transportation are required.

22 . Feed and stabling of horses (subrouchers required), ferriage and tolls.

23. The employment of guides when absolutely necessary. Subrouchers showing the necessity for a guide are required. 


\section{Charges for local or long-distance telephone} messages on public business will be allowed, provided a statement is furnished of the person and place communicated with, the time occupied, and substance of the message. When long-distance telephone is used, a subroucher must be furnished when practicable, except where an automatic pay station has been used. In the latter case the account must contain a statement to that effect.

\section{MISCELLANEOUS EXPENSES.}

25. Labor or other assistance should be specifically authorized by the Commisioner of Fisheries before such employment is engaged. Such assistance should not be paid for hy the field employee, but the account (voucher Form 433), covering same should be certified to in the usual manner and forwarded for settlement. Such account should show when the scrvice began and ended. If it is found that the payee will not wait for reimbursement in the regular inanner, payment may be made, receipt therefor being taken on a subvoucher. Such item in the account must be accompanied by full explanation. In no case can assistance be employed in Washington. 26. Miscellaneous supplies, repairs, and the like should have special authorization or they will not be allowed, and the extimated cost of such supplies or repairs should be submitted on requisition Form 762, before the purchase is made. Such supplies should not be paid for by the employee, but the account (voucher Form 810), covering the same should be certified to in the prescribed manner and forwarded ior settlement, unless the payee demand immediate payment, when the procedure may be followed as authorized in section 25 .

27. Articles of stationery, such as paper, pens, penholders, pencils, etc., should not be purchased. Such supplies should be obtained upon requisition on the Burreau of Fisheries. 
28. Freight and express.-Freight must always be shipped on government bills of lading and not paid for in cash. When goods are ordered the merchant or shipper should be supplied with a govermment hill of lading. Canceled bills of lading must be returned to the Dureau at once.

29. Prepaid express receipts presenterl as sulbvouchers must show weight, rate, and the rate for adrance charges.

30. Telegrams. - All employees will be furnishred with telegraph identification cards of both the Postal TelegraphCable Company and the Western Lnion Telegraph Company, which should be used in all cases where telegrams are sent on official business. Then these cards are used no payment at time of sending or receiving telegrams should be made. Where the employees of telesraph companies refuse to recognize telegraph identification cards, the tolls should be paid and a copy of the telegram, properly receipted for the amount paid, attached to the account to support the charge, and a statement made that telegraph identification cards were not recognized.

31. The telegraph should be resorted to as a means of communication only in cases of emergency, or when especially instructed to use that means of communication. If this rule is violated, the cost of the telegram will be charged against the employee responsible for sending the mesage.

32 . In preparing telegrams care should be observed that all unnecessary words, initials, and figures in the arldress and body, and also in the signature thereof, are omitted.

33. Expenses for telegrams relating to leave of absence, payment of salary and accounts, or any other matter of a personal nature, will not be allowed, and such telegrams must not be sent to the Bureau "collect."

34. Payments for telegrams can be made only at the rates shown in schedule prescribed by the PostmasterGeneral indicated in the following table. If for any reason a message can be transmitted cheaper at commercial rates, those rates should be used. 
Governinext Rates for Twenty Words and Multiples of TWenty, Togetier with T'olls for AdDITIONAL WORDS.

\begin{tabular}{|c|c|c|c|c|c|c|c|}
\hline \multirow[b]{2}{*}{ WORDS. } & \multirow[t]{2}{*}{ DAY } & \multicolumn{4}{|c|}{ MESSAGES WHEN RATE IS- } & \multicolumn{2}{|c|}{$\begin{array}{l}\text { NIGHT MES- } \\
\text { SAGES WHEN } \\
\text { DAY RATE IS- }\end{array}$} \\
\hline & & $\begin{array}{c}25 \\
\text { cents. }\end{array}$ & $\begin{array}{c}30 \\
\text { cents. }\end{array}$ & $\begin{array}{c}35 \\
\text { cents. }\end{array}$ & $\begin{array}{c}40 \\
\text { cents. }\end{array}$ & $\begin{array}{c}20,25, \\
\text { or } 30 \\
\text { cents } \\
\text { night } \\
\text { rate is- }\end{array}$ & $\begin{array}{l}35 \text { or } 40 \\
\text { cents } \\
\text { night } \\
\text { rate is }\end{array}$ \\
\hline 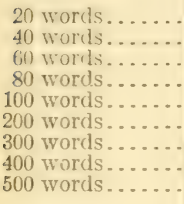 & $\begin{array}{r}30.20 \\
.40 \\
.60 \\
.80 \\
1.00 \\
2.00 \\
3.00 \\
4.00 \\
5.00\end{array}$ & $\begin{array}{r}\$ 0.25 \\
.50 \\
.75 \\
1.00 \\
1.25 \\
2.50 \\
3.75 \\
5.00 \\
6.25\end{array}$ & $\begin{array}{r}\$ 0.30 \\
.60 \\
.90 \\
1.20 \\
1.50 \\
3.00 \\
4.50 \\
6.00 \\
7.50\end{array}$ & $\begin{array}{r}\$ 0.35 \\
.70 \\
1.05 \\
1.40 \\
1.75 \\
3.50 \\
5.25 \\
7.00 \\
8.75\end{array}$ & $\begin{array}{r}\text { S0. } 40 \\
.80 \\
1.20 \\
1.60 \\
2.00 \\
4.00 \\
6.00 \\
8.00 \\
10.00\end{array}$ & $\begin{array}{r}\$ 0.15 \\
.35 \\
.55 \\
.75 \\
.95 \\
1.95 \\
2.95 \\
3.95 \\
4.95\end{array}$ & $\begin{array}{r}\$ 0.25 \\
.45 \\
.65 \\
.85 \\
.1 .05 \\
2.05 \\
3.05 \\
4.05 \\
5.05\end{array}$ \\
\hline Additional words. & & & & & & & \\
\hline 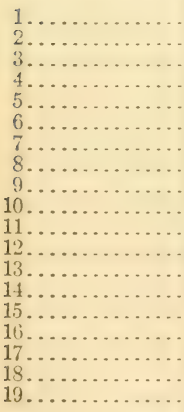 & $\begin{array}{l}.01 \\
.02 \\
.03 \\
.04 \\
.05 \\
.06 \\
.07 \\
.08 \\
.09 \\
.10 \\
.11 \\
.12 \\
.13 \\
.14 \\
.15 \\
.16 \\
.17 \\
.18 \\
.19\end{array}$ & $\begin{array}{l}.01 \\
.03 \\
.04 \\
.05 \\
.06 \\
.08 \\
.09 \\
.10 \\
.11 \\
.13 \\
.14 \\
.15 \\
.16 \\
.18 \\
.19 \\
.20 \\
.21 \\
.23 \\
.24\end{array}$ & $\begin{array}{l}.02 \\
.03 \\
.05 \\
.06 \\
.08 \\
.09 \\
.11 \\
.12 \\
.14 \\
.15 \\
.17 \\
.18 \\
.20 \\
.21 \\
.23 \\
.24 \\
.26 \\
.27 \\
.29\end{array}$ & $\begin{array}{l}.02 \\
.04 \\
.05 \\
.07 \\
.09 \\
.11 \\
.12 \\
.14 \\
.16 \\
.18 \\
.19 \\
.21 \\
.23 \\
.25 \\
.26 \\
.28 \\
.30 \\
.32 \\
.33\end{array}$ & $\begin{array}{l}.02 \\
.04 \\
.06 \\
.08 \\
.10 \\
.12 \\
.14 \\
.16 \\
.18 \\
.20 \\
.22 \\
.24 \\
.26 \\
.28 \\
.30 \\
.32 \\
.34 \\
.36 \\
.38\end{array}$ & $\begin{array}{l}.01 \\
.02 \\
.03 \\
.04 \\
.05 \\
.06 \\
.07 \\
.08 \\
.09 \\
.10 \\
.11 \\
.12 \\
.13 \\
.14 \\
.15 \\
.16 \\
.17 \\
.18 \\
.19\end{array}$ & $\begin{array}{l}.01 \\
.02 \\
.03 \\
.04 \\
.05 \\
.06 \\
.07 \\
.08 \\
.09 \\
.10 \\
.11 \\
.12 \\
.13 \\
.14 \\
.15 \\
.16 \\
.17 \\
.18 \\
.19\end{array}$ \\
\hline
\end{tabular}

NotE. - When the message contains less than 20 words charge is made for 20 words. 


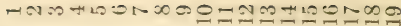

\begin{tabular}{|c|c|}
\hline •țnossțt & 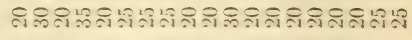 \\
\hline •Iddịss!̣s!̣t/ & 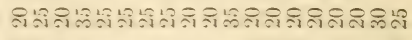 \\
\hline 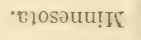 & เิติอง \\
\hline 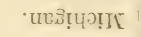 & 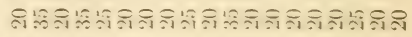 \\
\hline
\end{tabular}

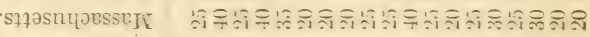

purtiut

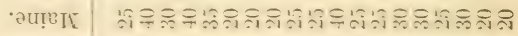

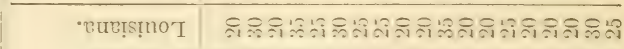

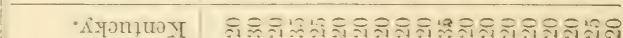

\begin{tabular}{|c|c|}
\hline -sustuey & 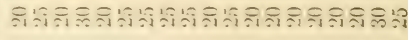 \\
\hline$\cdot$ e.11OI & 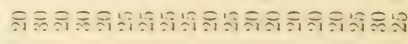 \\
\hline 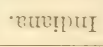 & 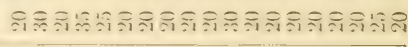 \\
\hline sṭom!lit & 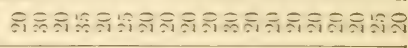 \\
\hline ००นใ? I & 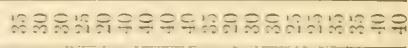 \\
\hline 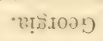 & 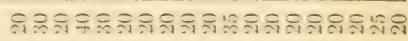 \\
\hline
\end{tabular}

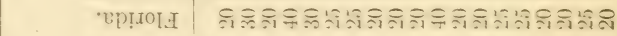

\begin{tabular}{|c|}
\hline आIIO) 7 ? \\
\hline
\end{tabular}

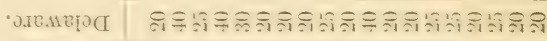

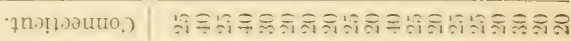

\begin{tabular}{|c|c|c|}
\hline & ope.10[0) & 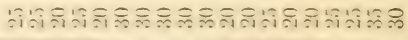 \\
\hline & 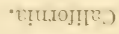 & 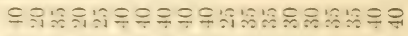 \\
\hline & 'stsury.iY & 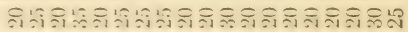 \\
\hline & 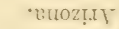 & 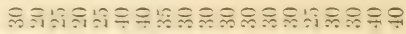 \\
\hline & - vuxquा & 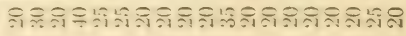 \\
\hline & 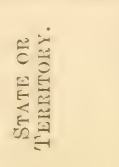 & 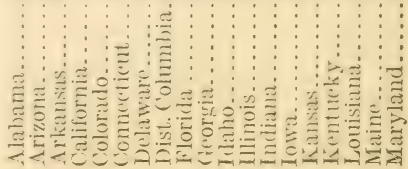 \\
\hline
\end{tabular}

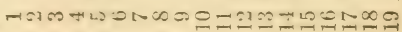




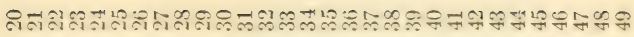

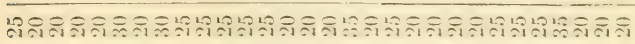

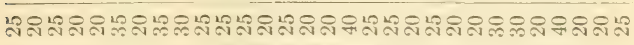

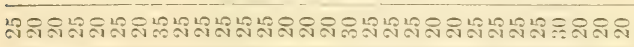

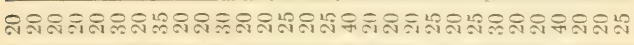

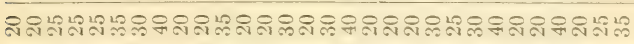

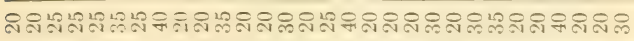

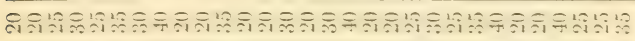

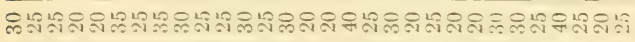

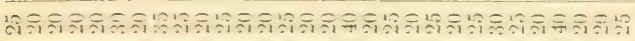

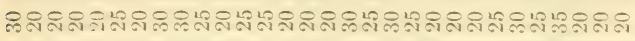

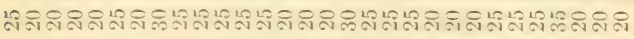

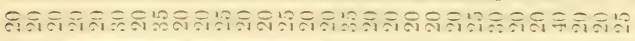

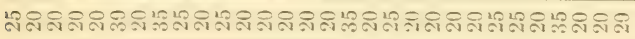

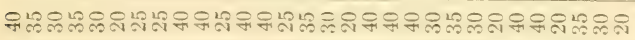

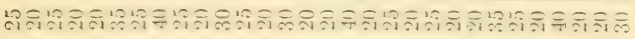

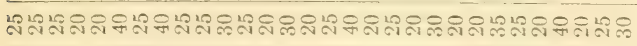

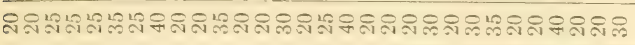

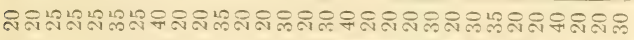

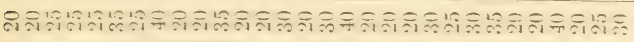

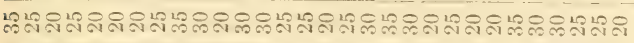

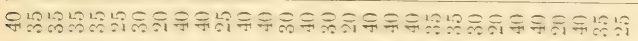

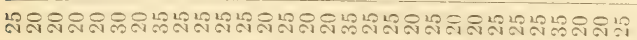

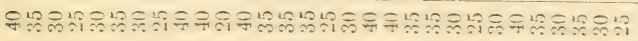

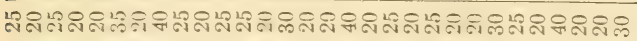

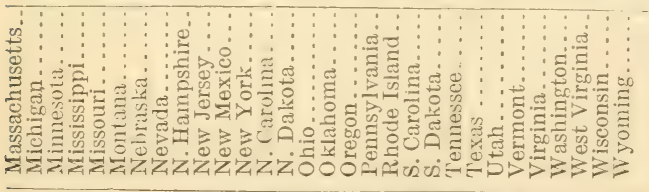

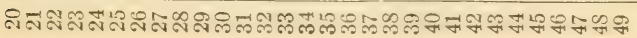




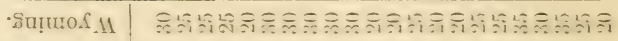

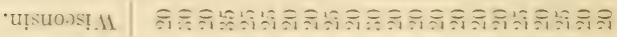

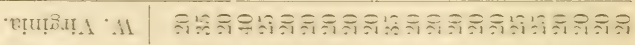

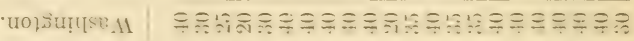

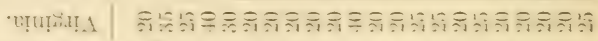

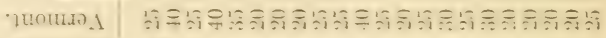

\begin{tabular}{|c|c|}
\hline$\cdot т[1.7$ & 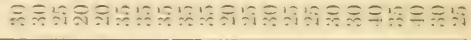 \\
\hline$\cdot$ StexDLL & ลิง \\
\hline 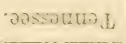 & 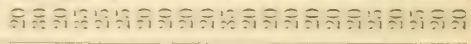 \\
\hline 'थ) & 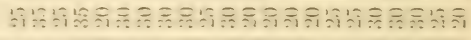 \\
\hline
\end{tabular}

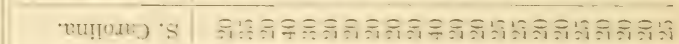

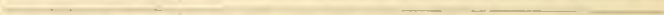

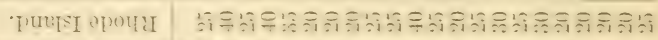

\begin{tabular}{|c|c|}
\hline 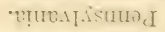 & 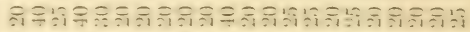 \\
\hline
\end{tabular}

ข

\begin{tabular}{|c|c|}
\hline 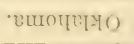 & 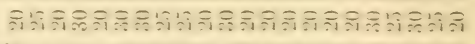 \\
\hline 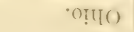 & 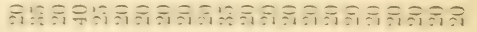 \\
\hline
\end{tabular}

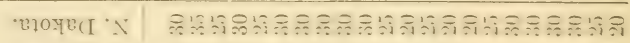

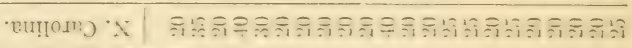

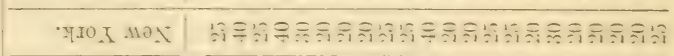

。

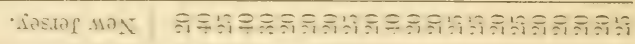

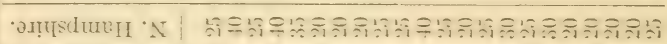

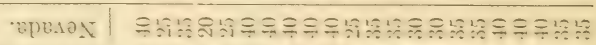

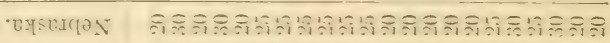

\begin{tabular}{|c|c|}
\hline$\therefore$ : : Eut? & 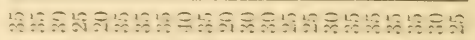 \\
\hline 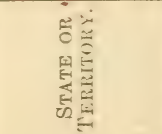 & 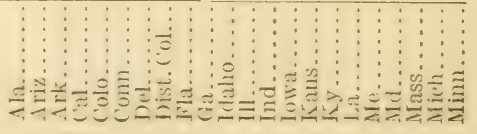 \\
\hline
\end{tabular}

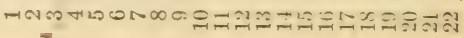




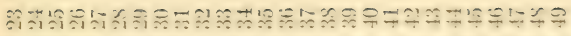

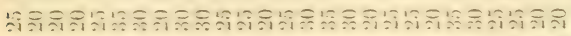

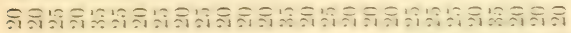

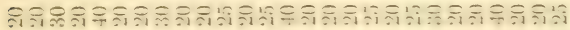

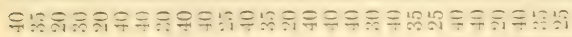

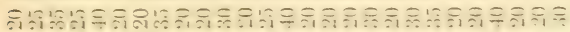

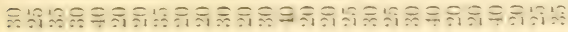

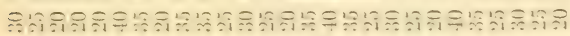

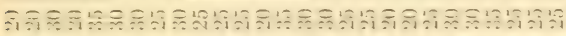

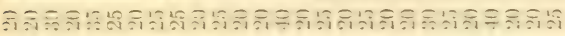

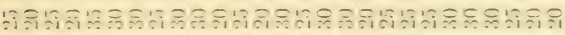

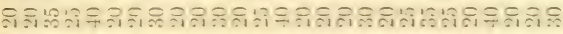

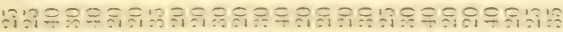

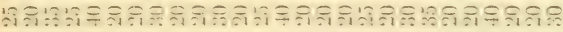

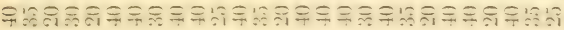

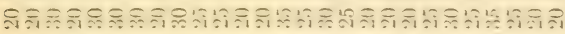

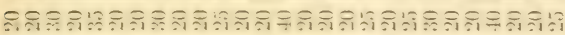

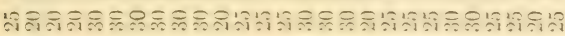

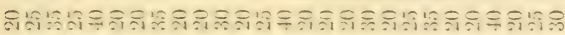

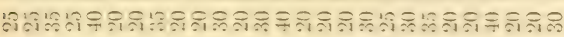

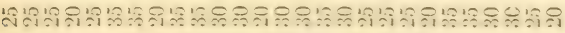

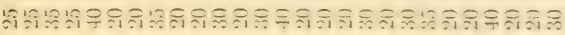

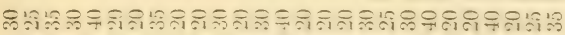

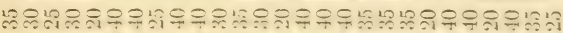

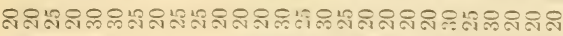

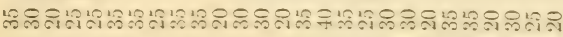

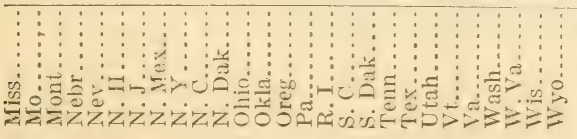

ศิ 


\section{Words to be counted and charged for.-In a} prepaid message the under-mentioned words will be counted and charged for, viz:

The address and signature.

All words in an extra date.

In the check, the words "Deliver and report charges," "Collect delivery charges," "Report delivery," and "Repeat back."

In collect messages the word "collect" in the check will be counted but will not be charged for.

When a messace bears two or more addresses, and delivery is to be made to each address, it will be chared for as two or more messages, as the case may be. A mexsage addressed, for example, to " $\| 1$. Brown, 197 Broadway,

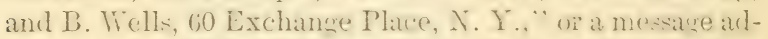
dresed, for example, to "W. Brown and B. liells, 1!n Broadway," will in each cate be charged for as two mes-

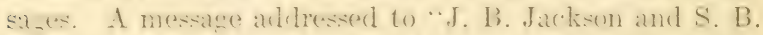
Sinith, Senate (hamber, Washington, D. '.," will be charged for at $t w 0$ messacess. The additional address will not be considered as extra words.

In counting a mesage, dictionary words, initial letters, surnames of persons, names of cities, fowns, rillages, States or Territories, or names of the canatian provinces, will be counted and charged for sarch as one word. The ahbreviations for the names of cities, towns, villages, States, Territories, and Provineses will loe connted and charged for the same as if written in full. In names oí comntries or counties all the words will be counted and charged for.

Abbreriations of weights and measures in common we will be counted each as a word.

-Tó prevent liability to eror and reduce cozt, numbers and amounts should be written in words.

In ordinil numbers the affixes st., d., nd., rd., and th. will each be counted as one word. 
All groups of letters, whether pronouncealile or not, when such groups are not dictionary words and are not combinations of dictionary words, will in domestic messages be counted at the rate of five letters or fractions of five letters to the word. Figures, decimal points, and hars of division will be counted, each separately, as one word.

The following example will illustrate the application of these rules:

Van Dorne

McGregor

O'Connor.

DeWitt

Brown. jr

New York (or N. Y.)..... 1

New York State......... 2

Nova Scotia (or N. S.).... 1

St. Louti................

East St. Louis............

North Carolina . . ......... 1

Queen Ann County....... 3

New Mexico............. 1 "

District of Columbia (or

D. C.).............. 1 "

North Amerira ......... 2

$44.42 \ldots \ldots \ldots \ldots \ldots \ldots . .5$

$42 \mathrm{~B} 618 \ldots \ldots \ldots \ldots \ldots \ldots 6$

$7 t_{1}^{3} \ldots \ldots \ldots \ldots \ldots \ldots \ldots$

No. 195 $2: 1$ st.......... s

10000000 .

Ten millions

3 il (or $3 \mathrm{r}$ l)
1 word

th..................... 3 word

Lbs

Cwt. . .

Hhds ...... "

Amaturecis............. " "

Adbantia............... 2 "

Chancin..................

Interavis............... 2 "

Byxtrm!3)............. is "

Xyf194sm............ \& "

All-right or alright....... 2 "

EXCEPTIONS.

A. M................ 1 "

P. MI................ I "

F. U. B. (or foh)........ 1 "

C. O. D. (or cod) ........ 1 "

C. I. F. or C. F. I. (or cif or (fi) $\ldots \ldots \ldots \ldots \ldots \ldots \ldots \ldots$

ก. K................ " 1

C. A. F. (or caf)......... 1 "

P'er cent................ "

PREPARATION OF ACCOUNTS.

See paragraphs $7,17,20,24,25,26$.

36. Accounts for travel expenses are to be rendered on Form 817 and its corresponding attached memorandum; and for purchases of material on Form $\$ 10$ and corresponding memorandum. Pay rolls covering the employment of temporary labor are to be rendered on Form 433, in 
duplicate, accompanied by report of temporary labor, Form 825, in triplicate.

37. Accounts should be transmitted to the Bureau through the officer immediately in charge. Letters transmitting accounts are unnecessary.

38. The address to which check is to be sent should always be indicated in the space provided for that purpose.

39. Not more than one account a month (excepting for distribution work) is to be rendered, and it must include all expenses for that month. It may also include items suspended from accounts for previous months.

40. Items suspended for explanation should be included as the last entry in the first regular monthly account submitted after the receipt of notice of suspension, and should be accompanied by the required explanation and all correspondence relating thereto.

41. Where items of expense are omitted, the regulations of the Treasury Department will not permit their being included in a subsequent account.

42. Accounts should be given an appropriate heading, stating the work engaged upon, and the points betreen which travel is performed, and be complete as to affidarits and the signatures to all certificates and other requirements indicated on the blanks.

43. Each account as submitted must have attached a memorandum, which should contain an exact transcript of all the items in the original. (See also par. 36.) No signatures should appear on this memorandum.

44. It is preferred that accounts be typewritten and the memorandum be a carbon copy. If a typewriter is not available, black ink should be used. Aniline inks, such as purple, violet, red, etc., should not be used. Proper ink will be supplied by the Bureau.

45. The entries should follow each other according to the order in which the expenses were incurred with the date of each, and all accounts should be accurately added and the total for each page carried from the bottom of that 
page to the top of the next succeeding page. The charges should be carried to the dollars and cents columns direct, so that two items will not appear on the same line. No entries should be made between the lines of the account.

46. The entries must show briefly the character of the expense in each case, in addition to the reference to the subvoucher; e. g., "Conveyance Washington to (hain Bridge," "Supplies," "Hardware," "Board and lodging," etc. If the charge is for board and lodging, the period must be shown. The place where the expense is incurred must always be given.

47. Travel accounts must show a complete itinerary. When transportation requests are used, the date of issue, points between which transportation is furnished, and the number of the request must be shown on the account. (See par. 8.) Free transportation must also be shown. When cash fare is paid, the initials of the road traveled must be given.

48. Travel accounts must be accompanied by original travel orders or copies thereof except when orders bear serial numbers. When copies of orders are furnished, they must be certified as "true copies." If the order bears a serial number, the number is to be inserted in the account in the proper space.

49. Every account and subvoucher must bear the signature of the payee in his own handwriting, and his postoffice address should be stated in the place indicated. The signature of the payee should correspond to the name of payee in every particular.

50. All items must be supported by subvouchers, or a satisfactory reason given, except for railroad tickets purchased at offices, porters' fees, baggage transfers, detached meals, and jurat fees. See also paragraphs 12, 13, $16,17,21,22,23,24,25,29$, and 30 .

51. Subvouchers should be numbered consecutively and entered in numerical order in the column prepared for the purpose in the account, and should be securely 
attached to the account in numerical series. They must be complete as to dates, amounts, etc.

52. Signatures by mark to subvouchers should be witnessed by some one other than the person rendering the account. and the witness's name should appear as such. When it is impossible to have signature by mark witnessed, the person rendering the account may act as witness, explaining why he did so.

53. Subvouchers must be stated in the name of the person, firm, company, or corporation rendering the service. If a firm, the receipt to the subvoucher must be in the usual firm simnature: if an incorporated or mincorporated company, the receipt musi be in the corporate name, folowed by the autoraph simature of the officer (with his title) authorized to receive moneys and receipt thereior. Where possible, subvouchers should be filled out by the payee. If that can not be dome. the amount in figures should be writen by the person sinnine the reeeipt.

54. Erasuresor alterations should he avoided. and when made shmuld the cortified to by the ofiicer administering the waths as having been made before final execution. The certificate should indicate the alteration or erasure made.

55. Paragraph 3 of Treasury Department ( ircular No. 52 of the year 1907 provides that all accounts claiming reimbursement for actual necessary expenses incurred in the periormance of official duty, or mileage where provided by law; shall be verified by affidavit.

56. A clerk of a tnited States court, a United States commissioner, or an officer or employee in the executive service of the Government who is also a notary is not permitted to receive a fee from an employee for administering an oath to an account, and a charge for such service will not be allowed to the employee.

57. Wheir an officer or employee of the department verifies a voucher by his oath, before a clerk of a Lnited States court or a United States commissioner, the officer or em- 
ployee making such oath should not pay the fee for such service. The ('omptroller of the Treasury, in a decision dated July 31, 1906, held that such fees should be included in the arcounts of relerks and commissioners and paid by the United States upon settling their accounts at the Treasury, as provided for by section S.)t, Revised Statutes.

58. The nfficial chararter of the officer administering the oath should be shown by the impress soul of his office or by the certificate of the proper wficial of the State or Territory in which the wath is administered, showing his authority to admini-ter oaths. The jurat should always show the renue of a magistrate.

59. () inly one jurat fee will be allowed. The jurat iee is to he charued as the last item in the account, and must not exceed the legal rate.

60. The leal fees which notaries public and justices of the peace of the various states and Territories are authorized by law to charge for taking aflidarits to accounts and aflixing their seals thereto are set forth in the following table:

\begin{tabular}{|c|c|c|}
\hline State or Territory. & $\begin{array}{l}\text { Notaries } \\
\text { public. }\end{array}$ & $\begin{array}{l}\text { Justices of } \\
\text { the peace. }\end{array}$ \\
\hline Alabama.... & $\$ 0.50$ & $\$ 0.25$ \\
\hline Alaska: & & \\
\hline iet ............................. & .5 & .30 \\
\hline nd third distriets..................... & .7 & .4 \\
\hline 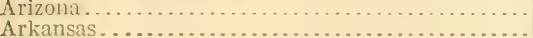 & .7 & $\begin{array}{l}.75 \\
.50\end{array}$ \\
\hline & .50 & .25 \\
\hline${ }_{\ldots \ldots \ldots \ldots \ldots \ldots \ldots \ldots \ldots \ldots \ldots \ldots \ldots \ldots \ldots \ldots \ldots \ldots \ldots}$ & 25 & .2 \\
\hline lecticut ............ & .3 & .10 \\
\hline Dela & .50 & .2 \\
\hline f Columbia................................. & .50 & .50 \\
\hline 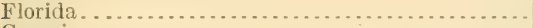 & .60 & .16 \\
\hline 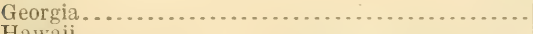 & .50 & .30 \\
\hline Hawaii . . . . . . . . . . . & .25 & 15 \\
\hline$\therefore \ldots \ldots, \ldots, \ldots, \ldots, \ldots$ & .25 & .15 \\
\hline na. & .50 & .25 \\
\hline 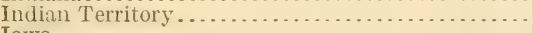 & $.2 \bar{j}$ & .25 \\
\hline 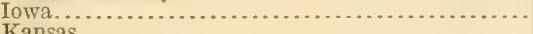 & .30 & .30 \\
\hline 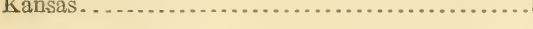 & & \\
\hline
\end{tabular}


State or Territory.

Kentuck $\nabla$

Louisiana.

Maine.

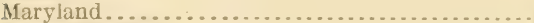

Mas.ichust'ts.

Michigan.

Minnesota.

Mississippi.

Missouri.

Montana

Nebraska.

Nevula.

New liampshire.

New Jersiry.

New Mexico.

New York.

North Carolina

Vorth Inakuta.

Ohio

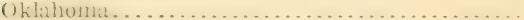

Oregon

I'ennstrini.

Except-

City of Philadelphia

Allwheny ('ominty.

Bedford, Berks, Blair, Cameron, Center, Clinton, Columbia, Dauphin, Delaware, Fulton, Lancaster, Lebanon, Luzerne, Lycoming, Montour, Northampton, Snyder, Somerset, Westmoreland, and W yoming counties.....

Erie, Schuylkill, and York counties...........

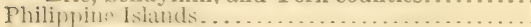
Rhorie Istand.

South Carolina.

South Dakota.

Tennessee.

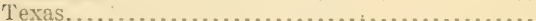

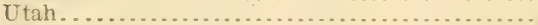

Vermont

Virginia

Washington

TVest I irgini:

Wisconsin

Wyoming.
Notaries Justices of public. the peace.

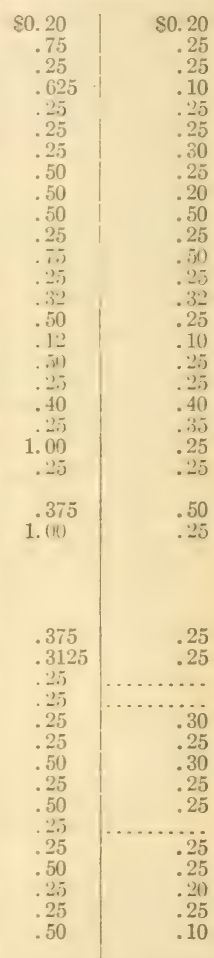

61. It has been held by the Treasury Department that a sर्रorn, aflidavit will not be required for expense accounts in this department where those incurring the expenses"are far removed from an officer authorized to administer oaths for general purposes, or where, by the making of such 
affidavit, the disclosure of the fact of the presence of the government officer, agent, or employer at the particular place where such aftidavit is taken will be detrimental to the public interests, or where, on account of the peculiar nature of the duties of the oflicer or employer, continuous travel is necessary without oppormunity for stops at places where the appearance before an officer authorized to administer oaths is possible "- provided, however, that such expense account-" shall be certified on honor as correct and accompanied by a statement of the conditions which make it impracticable to secure an afidarit."

61. All expense accounts not sworn 20 must contain specific explanations of the reasons why the oath is omitted, and must be certified to on honor in the space provided for the affidavit.

62. The following sample account has been printed to show the manner of preparation and to secure uniformity in entering the items of actual and necessary expenses. Being for the purpose of illustration only, it is not intended as an exhaustive enumeration of all proper expenditures. 


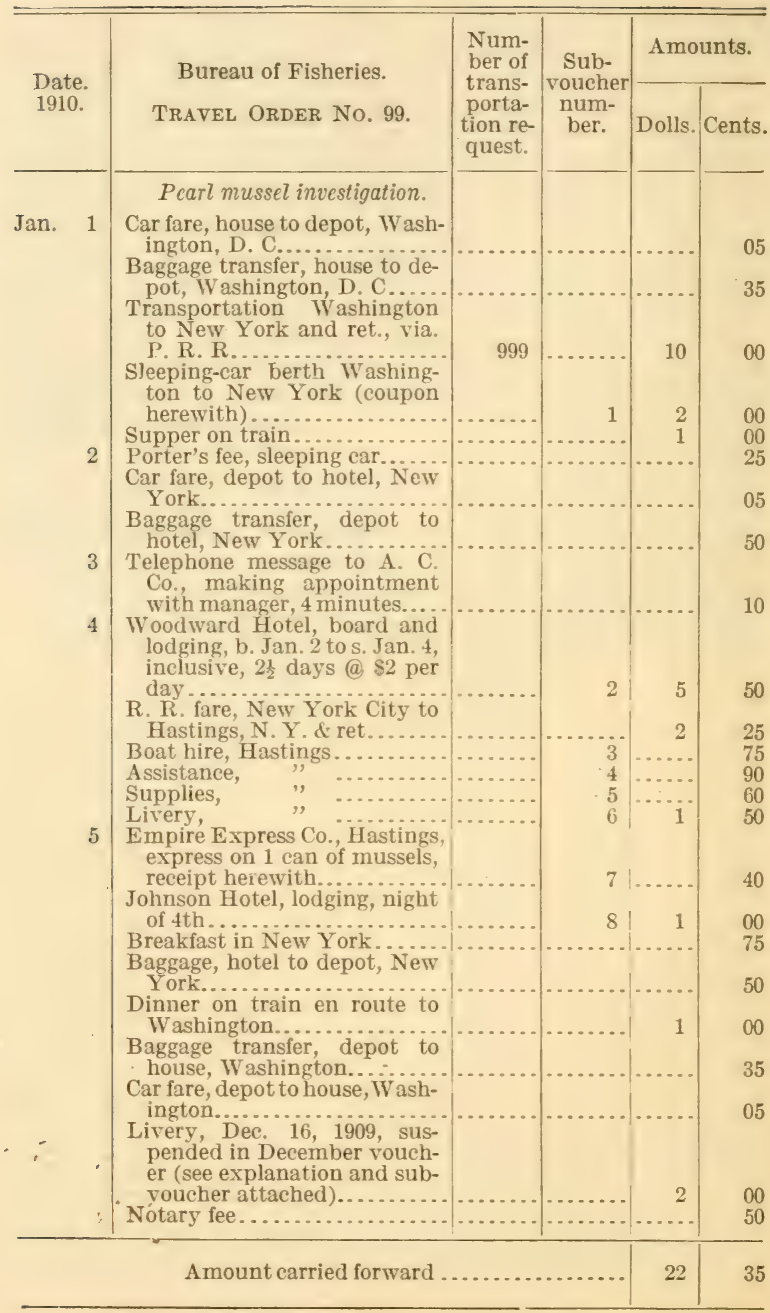


The following will give the general form of entries which should be made on subvoucher receipts, Form 527, which must accompany accounts when required by the regulations:

The United States to Woodward Hotel, Dr.

No. 2 .

\begin{tabular}{|c|c|c|c|}
\hline 1910. & $\begin{array}{l}\text { ARTICLES DELIVERED OR SERVICES } \\
\text { PERFORMED. }\end{array}$ & Dollars. & $\mathrm{Cts}$. \\
\hline Jan. 4 & $\begin{array}{l}\text { Board and lodging, breakfast Jan. } 2 \text { to supper } \\
\text { Jan. } 4 \text {, ine., } 2^{3} \text { days, at } \$ 2 \text { per day........... }\end{array}$ & 5 & 50 \\
\hline
\end{tabular}

RECEIVED at New York City, of John Smith, this 4 th day of Jan., 1910 , the full amount of the above account.

$\$ 5.50$.

WOODWARD HOTEL, Per JOHN WOODWARD,

Prop.

The United States to John Doe, Dr.

No. 3.

\begin{tabular}{|c|c|c|c|}
\hline 1910. & $\begin{array}{l}\text { ARTICLES DELIVERED OR SERVICES } \\
\text { PERFORMED. }\end{array}$ & Dollars. & Cts. \\
\hline Jan. 4 & $\begin{array}{c}\text { Boat hire, collecting mussels, } 3 \text { hrs., at } \\
25 \text { cts } \ldots \ldots \ldots \ldots \ldots \ldots \ldots \ldots \ldots \ldots \ldots \ldots \ldots\end{array}$ & & 75 \\
\hline
\end{tabular}

RECErved at New York City, of John Smith, this 4th day of Jan., 1910 , the full amount of the above account.

$\$ 0.75$.

JoHN DOE.

The United States to Geo. Johnson, Dr.

No. 4 .

\begin{tabular}{c|c|c|c|c}
\hline 1910. & $\begin{array}{c}\text { ARTICLES DELIVERED OR SERvices } \\
\text { PERFORMED. }\end{array}$ & Dollars. & Cts. \\
\hline Jan. 4 & Assistance collecting mussels, 3 hrs., at 30 cts. & $\ldots . . . .$. & 90 \\
\hline
\end{tabular}

RECEIvEd at New York City, of John Smith, this 4th day of Jan., 1910 , the full amount of the above account.

$\$ 0.90$.

Geo. Johnson. 
The United States to John Brown, Dr.

\begin{tabular}{|c|c|c|c|}
\hline 1910. & $\begin{array}{l}\text { ARTICLES DELIVERED OR SERTICES } \\
\text { PERFORMED. }\end{array}$ & Dollars. & Cts. \\
\hline Jan. 4 & 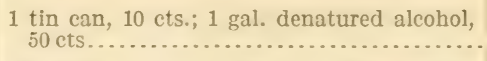 & & 60 \\
\hline
\end{tabular}

ReCerver at New York City, of John Smith, this 4th day of Jan., 1910 , the full amount of the above account.

$\$ 0.60$.

JOHN BROWN.

The United States to William A. Harris, Dr.

No. 6.

\begin{tabular}{l|c|c|c}
\hline 1910. & ARticles Delivered or services \\
PERformed. & Dollars. & Cts. \\
\hline Jan. $4 \mid \begin{array}{c}\text { Horse and buggy, one hour, driving from } \\
\text { Hastings mussel beds, in Hudson River. } \\
\text { No other means of conveyance.................. }\end{array}$ & 1 & 50 \\
\hline
\end{tabular}

Recerven at Hastings, $\therefore$. Y., of John Smith, this 4 th day of Jan., 1910 , the full amount of the above account.

$\$ 1.50$.

Williaji A. Harris.

The United States to Johnson Hotel, Dr.

No. 8 .

\begin{tabular}{l|c|r|r}
\hline 1910. & $\begin{array}{c}\text { ARticles Delivered or SERvices } \\
\text { PERFormed. }\end{array}$ & Dollars. & Cts. \\
\hline Jan. 5 & Lodging night of Jan. 4..................... & 1 & 00 \\
\hline
\end{tabular}

Recerved at New York, of John Smith, this 5th day of Jan., 1910, the full amount of the above account.

$\$ 1.00$.

JOHNSON HOTEL, Per M. MADISON, Clk. 


\section{RECAPITULATION.}

\section{EXPENSES WHICH IIAY BE ALLOWED.}

Fares:

Railroad and other, see par. 7, 8, 10, 11, 12.

Sleeping and parlor car, see par. 13.

Street car, see par. 15.

Baggage transfer, see par. 15.

Fees, see par. 14.

Hotel accommodations, see par. 16, 17, 20.

Board and lodging, see par. 17, 20.

Livery and special transportation, see par. 21, 22.

Guides, see par. 23.

Telegrams, see par. 30, 31, 32, 34, 35 .

Telephone messages, see par. 24.

Labor, see par. 25.

Supplies, see par. 26.

\section{EXPENSES WHICH NAY BE ALLOWED CONDITION-} ALIY.

Cabs and busses, see par. 15.

Fees, see par. 14.

\section{EXPENSES WHICH WILL NOT BE ALLOWED.}

Extra fares on limited trains, see par. 10.

Meals or lodging owing to avoidahle detention, see par. 18.

Meals or Indging at place of residence, sec par. 19.

Stationery, see par. 27.

Freight, see par. 28.

Telegrams of personal nature, see par. 33. 



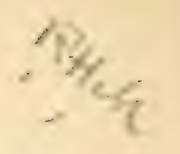






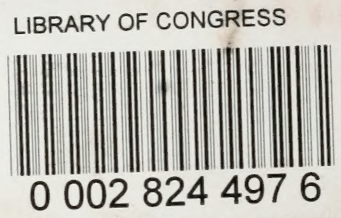

